\title{
Una mirada reflexiva sobre la educación y la gestión pública desde la transdisciplinariedad
}

\author{
Luis del Aguila Valencia \\ luisdelaguilav@gmail.com \\ https://orcid.org/0000-0002-8552-8626 \\ Escuela de Posgrado Universidad Cesar Vallejo \\ Juan Carlos Tuesta Hidalgo \\ https://orcid.org/0000-0002-9316-202X \\ Escuela de Posgrado Universidad Cesar Vallejo \\ Oscar Alejandro Tuesta Hidalgo \\ https://orcid.org/0000-0003-2231-9365 \\ Escuela de Posgrado Universidad Cesar Vallejo \\ Hipólito Percy Barbaran Mozo \\ pbarmozito@hotmail.com \\ https://orcid.org/0000-0002-9316-202X \\ Escuela de Posgrado Universidad Cesar Vallejo
}

\section{RESUMEN}

La transdisciplinariedad se define como una forma de organización de los conocimientos que trascienden las disciplinas más allá de su desarrollo aislado o fragmentado. En ese contexto, la gestión pública requiere de una reflexión para que descienda con más pertinencia en la solución de sus múltiples problemas, en la medida que promueva la integración de varias disciplinas en la formulación e implementación de las políticas públicas. Por eso, mediante esta revisión se pretende participar en el diálogo y discusión sobre la posibilidad de asumir la administración y gestión pública de la educación como un hecho complejo que articula una serie de conocimientos y prácticas transdisciplinares provenientes de la pedagogía y sus ciencias complementarias con el único propósito de comprender los problemas y plantear soluciones relacionadas con los fines de la educación pública. Se realizó un estudio bibliográfico exploratorio con enfoque cualitativo, se revisaron 12 artículos científicos, la búsqueda de la información se realizó en bases de datos como Scopus, Dialnet, Scielo y Redalyc, utilizando palabras clave como transdisciplinariedad, desarrollo educativo y desarrollo integral. El estudio concluye que, 
la educación es un derecho y es una responsabilidad del Estado garantizarlo en el marco de la gestión pública; dotándole una perspectiva transdisciplinar a la gestión, que articule e integre la convergencias de diferentes teorías y enfoques provenientes de las ciencias de la educación con la formación integral del ser humano y el desarrollo del territorio; por cuanto, la educación es un hecho social complejo que rebasa las fronteras de la pedagogía y demás ciencias auxiliares.

Palabras clave: transdisciplinariedad; desarrollo integral; gestión pública. 


\title{
A reflective look at education and public management from transdisciplinarity
}

\begin{abstract}
Transdisciplinarity is defined as a form of organization of knowledge that transcends disciplines beyond their isolated or fragmented development. In this context, public management requires reflection so that it descends with more relevance in the solution of its multiple problems, to the extent that it promotes the integration of various disciplines in the formulation and implementation of public policies. Therefore, through this review, it is intended to participate in the dialogue and discussion about the possibility of assuming the administration and management of public education in education as a complex fact that articulates a series of transdisciplinary knowledge and practices from pedagogy and its complementary sciences with the sole purpose of understanding the problems and proposing solutions related to the purposes of public education. An exploratory bibliographic study with a qualitative approach was carried out, 12 scientific articles were reviewed, the information search was carried out in databases such as Scopus, Dialnet, Scielo and Redalyc, using keywords such as transdisciplinarity, educational development and integral development. The study concludes that education is a right and it is the responsibility of the State to guarantee it within the framework of public management; endowing management with a transdisciplinary perspective, which articulates and integrates the convergences of different theories and approaches from educational sciences with the integral formation of the human being and the development of the territory; because, education is a complex social fact that goes beyond the borders of pedagogy and other auxiliary sciences.
\end{abstract}

Keywords: transdisciplinarity; integral development; public management.

Artículo recibido: 05 octubre. 2021 Aceptado para publicación: 02 noviembre 2021 Correspondencia: luisdelaguilav@gmail.com Conflictos de Interés: Ninguna que declarar 


\section{INTRODUCCIÓN}

El presente artículo se realiza con la finalidad de revisar la mayor cantidad de información posible sobre el complejo tema de la Transdisciplinariedad y su relación con el desarrollo integral de la persona, a través de la educación desde la perspectiva de la nueva gestión pública; se pretende registrar, sistematizar y organizar la información y utilizarla a favor del desarrollo de una propuesta en el campo de la educación que repercuta positivamente en la sociedad, ya que el desarrollo personal, social e institucional, en el mundo actual, se ha vuelto cada vez más complejo en todas sus dimensiones, lo cual ha convertido lo metodológico en procesos complicados y difíciles, restando su eficacia para conocer en profundidad la realidad a la cual citamos y para lo cual necesitamos conocimientos que ayuden a lograr el progreso de la sociedad en que vivimos(Gedeón \& García 2009). La nueva gestión pública ha penetrado con fuerza en la agenda educativa en los últimos años con lo cual se busca aplicar conocimientos e instrumentos de la gestión empresarial y de disciplinas relacionadas que buscan mejorar la eficiencia, la eficacia y el rendimiento general de los servicios públicos en las burocracias modernas (Verger \& Norman 2015), para ello es necesario crear una mejor ciencia, en el sentido de que sea capaz de abordar los problemas humanos fundamentales y, también, aquélla capaz de ser juzgada en base a criterios ético-políticos vinculados a las necesidades sociales de los pueblos (Rodríguez \& Leonidas, 2011); asimismo buscar la unificación de la ciencia, buscar relaciones que las trasciendan para integrarlas en un conjunto con sentido, que busque no solo el cruzamiento e interpenetración de las disciplinas sino que borre los límites que existen entre ellas para integrarlas en un sistema único (Peñuela, 2005), esto alcanzaría al campo educativo en donde vemos serias falencias que necesariamente deben ser abordadas desde una nueva propuesta orientada hacia la transdisciplinariedad. Desde inicios de la década del ochenta del siglo pasado, surgieron diversos métodos, estrategias, procedimientos, técnicas e instrumentos, principalmente en el campo de las ciencias humanas, que buscan abordar y enfrentar esta realidad. Estos procesos metodológicos se conocen hoy día con el nombre general de metodologías cualitativas, y han sido divulgados en un alto número de publicaciones, que van desde unos 400 libros hasta más de 3500 publicaciones parciales (capítulos de libros y artículos de revistas (Martínez, 2012). Estas orientaciones metodológicas tratan de responder a la complejidad de las realidades de la vida moderna $\mathrm{y}$, al mismo tiempo, tener procedimientos rigurosos, sistemáticos y críticos, es decir, 
poseer una alta respetabilidad científica, lo cual puede lograrse sobre la base de transdisciplinariedad (Ibídem). Edgar Morin, define la transdisciplinariedad como una visión del mundo que busca ubicar al hombre y a la humanidad en el centro de nuestra reflexión, y desarrollar una concepción integradora del conocimiento. Para ello, esta corriente de pensamiento ha desarrollado tres pilares: los niveles de realidad, la lógica del tercero incluido y la complejidad, a partir de los cuales pretende fundar una metodología que aborde la cuestión humana y del conocimiento desde una perspectiva de interconexión en el sentido de complexus o conocido como "lo que está tejido junto" (Bierbrauer \& Márquez, 2013). La búsqueda del conocimiento a través de la pedagogía nos obliga a dedicarnos al nutrimento de las visiones y conceptualizaciones y al discernimiento de los enfoques que se nos presentan actualmente en las políticas públicas y académicas institucionales: tal es el caso de la transdisciplinariedad. Para comprender mejor, es importante hacer algunas precisiones $y$ diferenciaciones entre multidisciplinariedad, interdisciplinariedad y transdisciplinariedad. La multidisciplinariedad y la interdisciplinariedad fueron tratadas y estudiadas por varios autores, así, se precisa que etimológicamente multidisciplinariedad significa muchas disciplinas, es decir, varias disciplinas abordando el mismo objeto de estudio, sin embargo en este abordaje las disciplinas no están conectadas entre sí y cada una estudia el tema de manera aislada. Por su parte la interdisciplinariedad, es la relación o integración entre disciplinas. Esta integración o intercambio entre disciplinas es de naturaleza intrínseca de sus métodos, o sea, al momento de abordar un problema u objeto de estudio sus métodos son comunes, de una u otra área del conocimiento (Varona, 2005). La transdisciplinariedad tiene contenidos de amplia y compleja significación y en poco tiempo no se ha podido acumular teoría y práctica que permitan definir el término sin minimizarlo o sobredimensionarlo, en tal sentido se corren riesgos en la definición y argumentación de propuestas que nos acerquen a ese objetivo (Carmona, 2004). Esta insuficiencia de teorías en el estudio de la transdisciplinariedad nos conduce a una crisis paradigmática, que afecta todas nuestras relaciones con la vida, incluyendo la escuela. En tal sentido, la problemática actual requiere del concurso de educadores que tengan un panorama teórico más amplio, fundamentado por la transdisciplinariedad nutrida por la complejidad, para que podamos encontrar soluciones de acuerdo a la complejidad de la problemática. En este sentido, y para poder realizar la tarea educativa, es importante que 
se avance por los caminos de la ciencia y que haya apertura al conocimiento, porque se necesita que exista claridad ontológica y epistemológica para entender mejor cómo se manifiesta la realidad y como se realizan los procesos de construcción del saber y del aprendizaje (Cândida, 2010). El pensamiento transdisciplinar conecta la ontología, epistemología y la metodología, trayendo nuevas bases para la renovación filosófica y educativa al dar prioridad a las relaciones, a las interacciones, a las emergencias, a las redes y a sus procesos auto-eco-organizadores, dialógicos, recursivos y emergentes, con lo cual queda claro que en vez de usarse la lógica binaria excluyente, tenemos que trabajar a partir de una lógica ternaria que une o que aparenta estar desunida, así como del reconocimiento de los niveles de realidad existentes y de la complejidad presente en la realidad (Martínez, Ortiz \& González, 2007) . La transdisciplinariedad, como principio epistemológico, implica una actitud de apertura con la vida y de todos sus procesos, actitud que ayuda a sobrepasar las barreras disciplinares en el intento de entender mejor lo que está más allá de los límites y de las fronteras establecidas (Cevallos, 2017). Un principio que requiere que el pensamiento vaya más allá de los aspectos establecidos de los cognitivos fundamentados en el desarrollo de competencias y de habilidades para que el proceso pueda de verdad hacer eco en la subjetividad humana. Estudiosos como Bateson, Foerster, Heisenberg, Göel, Kunh, Maruyama, Nicolescu, Prigogine, Shannon, entre otros, hicieron planteamientos sobre los principios gnoseológicos del pensamiento complejo que sirvieron de base para fundamentar la transdisciplinariedad. Estos autores han contribuido al conocimiento desde diversas perspectivas, pero en el caso específico de la transdisciplinariedad, se destaca a Basarab Nicolescu, que es uno de los más importantes defensores de este concepto, en su libro Transdisciplinarity: Theory and Practice plantea generar un marco de pensamiento que integre análisis científicos, sociales, culturales y espirituales, con la finalidad no sólo de comprender el mundo presente sino también la modelización de su evolución (Jurado, 2013). Para Nicolescu, la palabra transdisciplinariedad es derivada de la interdisciplinariedad, pero diferenciada de ésta (Cândida, 2010).

En el ámbito educativo, la transdisciplinariedad debe ser enfocada desde su dimensión política, económica, cultural, social, formativa, científica, filosófica, etc. y enfrentar el reto de educar adecuadamente al ser humano desde la concepción (Espinoza, 2011); es decir, formar a la persona de manera global, integral; esto significa formar sus emociones, 
sentimientos, a enfrentar las incertidumbres propuestas por Morín, temores o sus demonios internos, como diría Goethe (a través de la psicología); formar su pensamiento a través de la lógica, la matemática; formar o practicar valores a través de la ética, que ha venido a menos en estos últimos años; a formar o educar en la toma de decisiones sociales, o el poder colectivo a través de la Politología; formar su habilidad comunicativa a través del Lenguaje o la Lingüística; formar su valoración hacia los demás, para aprender a convivir y a tolerar otras culturas, costumbres, a valorar su propia cultura a través de las ciencias sociales (Antropología, Etnología, Historia); formar su comunicación con otras culturas a través del aprendizaje de otros idiomas; formar su capacidad de selección y búsqueda de información a través de la tecnología, pero con dominio de procesos metodológicos científicos (Gonzáles, 2014). A partir de esto, se concretará la formación de un ser integral que responda a una sociedad de características complejas concreta y específica, para mejorar las condiciones de vida del ser humano y erradicar la segmentación y la exclusión. El tránsito hacia la transdisciplinariedad en el campo de la educación implica una gestión diferente del territorio, es decir ver las políticas públicas desde las demandas ciudadanas y las características del territorio, lo cual solo puede lograrse desde la una Gestión Pública transdisciplinaria como un paradigma de futuro (Gutiérrez, 2017). Además, la nueva gestión pública, desde su visión moderna, tiene que formar estructuras multidimensionales que sirvan para articular de manera discutida y consensuada, la asociación de varios aspectos, desde la transdiciplinariedad y complejidad sobre la base del pensamiento complejo (López, 2017), esto supone trascender más allá de la combinación de modelos de gestión, es decir, por ejemplo, considerar a las organizaciones como un sistema y no a la organización con varios sistemas, fundamentado en la transdisciplinariedad (Valois, 2015). Sin duda, estamos frente al avance indetenible de la ciencia transdiciplinaria, que construye conocimiento libertador, no doctrinario ni segmentado; la transdisciplina implica un salto epistémico, en la que todos los caminos llevan al arte de tener como constante el factor humano y su condición, como una verdad multifacética, multilineal, multicultural (Gutiérrez, 2017). La realidad actual exige además “desfragmentar" los saberes, que se enseñe a partir de problemas interdisciplinarios; es necesario precisar que en la actualidad cualquier especialidad que no sabe colaborar con otras no es más que un "saber ciego", el diálogo 
transdisciplinario permite la complementariedad entre las ciencias y enriquece el conocimiento (Alfaro, Fernández \& Gonzáles, 2015).

\section{ESTRATEGIAS METODOLÓGICAS}

Para elaborar el presente artículo se utilizó la técnica de revisión bibliográfica, tomándose como referencia tesis doctorales referidas al tema y principalmente artículos científicos, además de otras fuentes de consulta como internet, revistas y libros. Con la finalidad de hacer algunas precisiones sobre el material bibliográfico se recurrió al método inductivo, además se ha realizado un análisis de tipo cualitativo, ya que se recopiló, revisó y sistematizó información a partir de una muestra de 12 artículos científicos relacionados a la gestión pública, la transdisciplinariedad y el desarrollo integral. Para estudiar de forma más precisa y minuciosa se utilizó el análisis sistemático, asimismo se hizo un diseño descriptivo, toda vez que se ha precisado las variables de acuerdo al contexto, para proponer un modelo de educación orientado a solucionar las deficiencias existentes actualmente.

\section{RESULTADOS Y DISCUSIÓN}

\section{Resultados.}

La revisión bibliográfica dio como resultado el hallazgo de una variedad de artículos relacionados con la transdisciplinariedad, la multidisciplinariedad, orientados al campo de la educación superior y la educación ambiental y muy poco a la educación básica, se revisaron también artículos referidos a la gestión; todos pueden ser considerados referentes para una reflexión sobre la gestión y la educación pública desde la transdisciplinariedad.

\begin{tabular}{|c|c|c|c|c|}
\hline $\mathbf{N}^{\circ}$ & Autor & País & Clasificación & Conclusiones \\
\hline 01 & $\begin{array}{c}\text { Gedeón y } \\
\text { García.(2009) }\end{array}$ & Venezuela & $\begin{array}{l}\text { Artículo } \\
\text { científico }\end{array}$ & $\begin{array}{l}\text { La educación transdisciplinaria } \\
\text { esclarece de una forma nueva la } \\
\text { necesidad de una educación } \\
\text { permanente, su finalidad es la } \\
\text { comprensión del mundo presente, } \\
\text { a través del conocimiento. La } \\
\text { disciplinariedad, } \\
\text { pluridisciplinariedad, la } \\
\text { interdisciplinariedad y la }\end{array}$ \\
\hline
\end{tabular}




\begin{tabular}{|c|c|c|c|c|}
\hline $\mathbf{N}^{\circ}$ & Autor & País & Clasificación & Conclusiones \\
\hline & & & & $\begin{array}{l}\text { transdisciplinariedad, se juntan } \\
\text { para lograr el conocimiento cada } \\
\text { vez más complejo de la realidad } \\
\text { subyacente. La educación } \\
\text { transdisciplinaria se funda en la } \\
\text { reevaluación del rol de la } \\
\text { intuición, } \\
\text { sensibilidad y el cuerpo en la } \\
\text { transmisión del conocimiento. }\end{array}$ \\
\hline 02 & $\begin{array}{c}\text { Verger \& } \\
\text { Norman(2015) }\end{array}$ & Brasil & $\begin{array}{l}\text { Artículo } \\
\text { Científico }\end{array}$ & $\begin{array}{l}\text { Los discursos e instrumentos de } \\
\text { la nueva Gestión Pública están } \\
\text { por todo el mundo, pero son } \\
\text { reformulados y traducidos en } \\
\text { contextos nacionales y locales de } \\
\text { tal manera que las políticas } \\
\text { finalmente retenidas pueden tener } \\
\text { aproximaciones } \\
\text { diferenciadas. En tal sentido, a la } \\
\text { hora de adoptar la nueva gestión } \\
\text { pública, algunos países hacen } \\
\text { más hincapié en el mercado y la } \\
\text { privatización, mientras que otros } \\
\text { están más preocupados por la } \\
\text { descentralización y la rendición } \\
\text { de cuentas. Esto claramente } \\
\text { demuestra que en el marco de la } \\
\text { nueva gestión pública es posible } \\
\text { abordar la gestión de un nuevo } \\
\text { currículo como parte de los } \\
\text { cambión necesarios a realizarse } \\
\text { adoptando }\end{array}$ \\
\hline
\end{tabular}




\begin{tabular}{|c|c|c|c|c|}
\hline $\mathbf{N}^{\circ}$ & Autor & País & Clasificación & Conclusiones \\
\hline & & & & $\begin{array}{l}\text { transdisciplinarias necesarias } \\
\text { para alcanzar el conocimiento y el } \\
\text { pensamiento complejo, esto a su } \\
\text { vez requerirá que los docentes } \\
\text { sean consultados cuando se } \\
\text { definen los estándares de calidad } \\
\text { y los criterios de evaluación de } \\
\text { sus estudiantes en pruebas } \\
\text { estandarizadas. }\end{array}$ \\
\hline 03 & $\begin{array}{c}\text { Martínez } \\
\text { (2012) }\end{array}$ & Venezuela & $\begin{array}{l}\text { Artículo } \\
\text { Científico }\end{array}$ & $\begin{array}{l}\text { En la actualidad es necesario que } \\
\text { cada disciplina haga una revisión, } \\
\text { reformulación o redefinición de } \\
\text { sus estructuras lógicas } \\
\text { individuales, establecidas aislada } \\
\text { e independientemente del sistema } \\
\text { total con que interactúan, ya que } \\
\text { sus conclusiones, en la medida en } \\
\text { que hayan cortado los lazos de } \\
\text { interconexión con el sistema } \\
\text { global de que forman parte, serán } \\
\text { parcial totalmente } \\
\text { inconsistentes. Las diferentes } \\
\text { disciplinas deberán, buscar y } \\
\text { seguir los principios de } \\
\text { inteligibilidad que se derivan de } \\
\text { una racionalidad más respetuosa } \\
\text { de los diversos aspectos del } \\
\text { pensamiento, una racionalidad } \\
\text { múltiple que, a su vez, es } \\
\text { engendrada por un paradigma de } \\
\text { la complejidad. En tal sentido, la }\end{array}$ \\
\hline
\end{tabular}


Una mirada reflexiva sobre la $\ldots$

\begin{tabular}{|c|c|c|c|c|}
\hline $\mathbf{N}^{\circ}$ & Autor & País & Clasificación & Conclusiones \\
\hline & & & & $\begin{array}{l}\text { transdisciplinariedad puede } \\
\text { definirse como un conocimiento } \\
\text { superior emergente, fruto de un } \\
\text { movimiento dialéctico de retro- y } \\
\text { pro-alimentación } \\
\text { pensamiento, que permite ir más } \\
\text { allá de los límites de diferentes } \\
\text { áreas del conocimiento } \\
\text { disciplinar y crear imágenes de la } \\
\text { realidad más completas, más } \\
\text { integradas y más verdaderas. }\end{array}$ \\
\hline 04 & $\begin{array}{c}\text { Bierbrauer \& } \\
\text { Márquez, } \\
\text { (2013). }\end{array}$ & Argentina & $\begin{array}{l}\text { Artículo } \\
\text { científico }\end{array}$ & $\begin{array}{l}\text { Los resultados de la } \\
\text { implementación de la propuesta } \\
\text { de trabajo transdisciplinario en el } \\
\text { aula fueron satisfactorios ya que } \\
\text { se superaron las expectativas } \\
\text { planteadas. Existen cuestiones } \\
\text { para optimizar y modificar para } \\
\text { próximas implementaciones de } \\
\text { este tipo de proyectos. En este } \\
\text { tipo de propuestas los docentes, } \\
\text { deben preocuparse por brindar } \\
\text { tareas orientadas por un lado a } \\
\text { promover lecturas y por otro, la } \\
\text { reflexión a partir de otros } \\
\text { lenguajes, además de generar } \\
\text { tareas } \\
\text { colaborativas, y actividades } \\
\text { actividades de intercambio para } \\
\text { generar conocimiento a partir de } \\
\text { las distintas intervenciones y co- }\end{array}$ \\
\hline
\end{tabular}




\begin{tabular}{|c|c|c|c|c|}
\hline $\mathbf{N}^{\circ}$ & Autor & País & Clasificación & Conclusiones \\
\hline & & & & $\begin{array}{l}\text { construir conocimiento. } \\
\text { modelo inter y transdisciplinario } \\
\text { genera una modalidad altamente } \\
\text { productiva y eficaz para el trabajo } \\
\text { a partir de proyectos en la escuela. } \\
\text { Los docentes deben poner a los } \\
\text { estudiantes en contacto con el } \\
\text { máximo número } \\
\text { oportunidades, enfrentarlo con } \\
\text { los diversos niveles de saber y } \\
\text { con las situaciones que son, o } \\
\text { simulan, futuras actividades } \\
\text { profesionales, esto contribuye al } \\
\text { desarrollo integral para la vida y } \\
\text { el trabajo de los alumnos. }\end{array}$ \\
\hline 05 & Varona (2005) & Cuba & $\begin{array}{l}\text { Artículo } \\
\text { científico }\end{array}$ & $\begin{array}{l}\text { La transdisciplinariedad es } \\
\text { resultado del desarrollo de la } \\
\text { ciencia y la tecnología y de las } \\
\text { conexiones, interacciones, } \\
\text { fusiones e integración de los } \\
\text { diversos planos de la vida } \\
\text { humana recogidas en el concepto } \\
\text { globalización. No constituye un } \\
\text { hecho espontáneo, ya que es } \\
\text { indispensable la acción } \\
\text { consciente del hombre. Toda } \\
\text { sociedad debe arribar a la } \\
\text { transdisciplinariedad, partiendo } \\
\text { de los centros de educación } \\
\text { superior, quienes tienen el papel } \\
\text { básico, ya que en la educación }\end{array}$ \\
\hline
\end{tabular}


Una mirada reflexiva sobre la $\ldots$

\begin{tabular}{|c|c|c|c|c|}
\hline $\mathbf{N}^{\circ}$ & Autor & País & Clasificación & Conclusiones \\
\hline & & & & $\begin{array}{l}\text { superior existen mejores } \\
\text { condiciones para } \\
\text { transdisciplinariedad, } \\
\text { embargo, es necesaria una labor } \\
\text { consciente encaminada a su } \\
\text { logro, lo cual debe tener su punto } \\
\text { inicial en el diálogo académico } \\
\text { entre los estudiantes de distintas } \\
\text { especialidades, incluidas las que } \\
\text { aparentemente no tienen nada } \\
\text { común entre sí. }\end{array}$ \\
\hline 06 & $\begin{array}{c}\text { Carmona, } \\
\text { (2004) }\end{array}$ & Venezuela & $\begin{array}{l}\text { Artículo } \\
\text { científico }\end{array}$ & $\begin{array}{l}\text { La estructura académica debe ser } \\
\text { el eje de la matriz generadora de } \\
\text { los cambios, para insertar la } \\
\text { teorización de los conceptos } \\
\text { pedagógicos en términos } \\
\text { interdisciplinarios } \\
\text { transdisciplinarios, de esta } \\
\text { manera se logrará generar una } \\
\text { ruptura con la super especialidad } \\
\text { o especialización de la } \\
\text { especialidad y se alcanzará } \\
\text { visiones más amplias y generales } \\
\text { de los proyectos complejos. Sin } \\
\text { embargo, los estudios en muchas } \\
\text { universidades no son asumidos } \\
\text { como un sistema, por lo que se } \\
\text { debe emprender la visión } \\
\text { sistemática como fundamento } \\
\text { relevante a fin de proponer la } \\
\text { inclusión }\end{array}$ \\
\hline
\end{tabular}




\begin{tabular}{|c|c|c|c|c|}
\hline $\mathbf{N}^{\circ}$ & Autor & País & Clasificación & Conclusiones \\
\hline & & & & 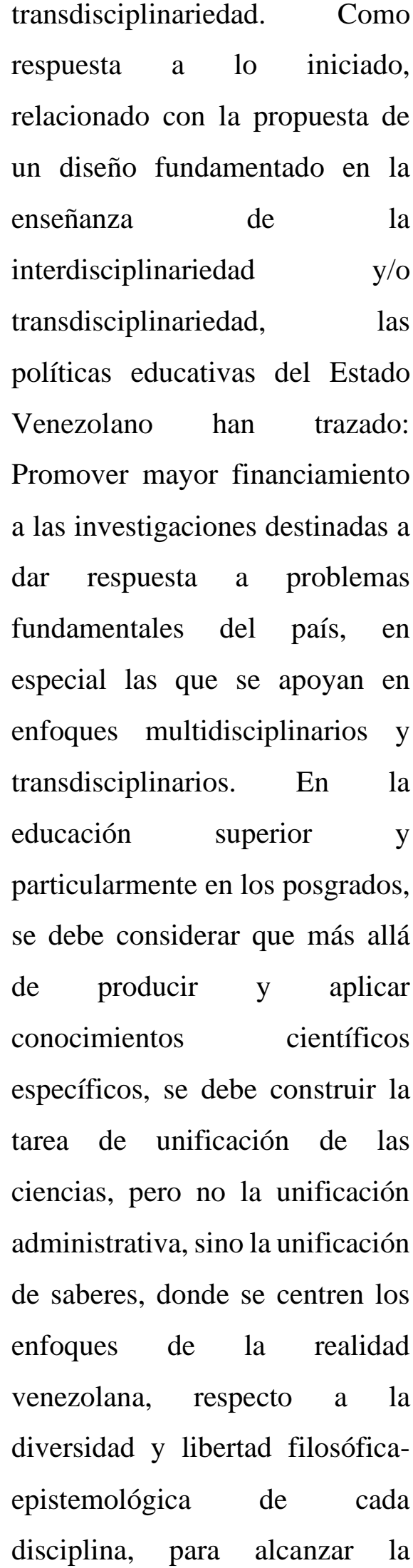 \\
\hline
\end{tabular}


Una mirada reflexiva sobre la $\ldots$

\begin{tabular}{|c|c|c|c|c|}
\hline $\mathbf{N}^{\circ}$ & Autor & País & Clasificación & Conclusiones \\
\hline & & & & $\begin{array}{l}\text { transdisciplinariedad como } \\
\text { propuesta para el avance de la } \\
\text { educación superior. }\end{array}$ \\
\hline 07 & $\begin{array}{c}\text { Cándida } \\
\text { (2010). }\end{array}$ & Brasil & $\begin{array}{l}\text { Artículo } \\
\text { científico }\end{array}$ & $\begin{array}{l}\text { La transdisciplinariedad ayuda a } \\
\text { evitar el pensamiento } \\
\text { determinista y reduccionista, la } \\
\text { planificación positivista y la } \\
\text { metodología que fragmenta la } \\
\text { realidad, el conocimiento y la } \\
\text { vida. Asimismo, evita, cualquier } \\
\text { tipo de relación dicotómica y } \\
\text { formalismo didáctico en la } \\
\text { escuela. Ya no es posible aceptar } \\
\text { la escuela reproductora del } \\
\text { conocimiento linear, } \\
\text { predeterminado y un discurso } \\
\text { pedagógico pobre y autoritario; } \\
\text { tampoco se puede seguir } \\
\text { sobrevalorando el método } \\
\text { didáctico como un modelo } \\
\text { abstracto y formal que } \\
\text { supuestamente quiere poner } \\
\text { orden en todas las unidades del } \\
\text { conocimiento, ya que la } \\
\text { reproducción del conocimiento y } \\
\text { las prácticas de instrucción no } \\
\text { combinan con los pensamientos } \\
\text { complejos y transdisciplinares } \\
\text { que requieren un nuevo diálogo, } \\
\text { nuevas aperturas, un pensamiento } \\
\text { más reflexivo y constructivo; esto }\end{array}$ \\
\hline
\end{tabular}




\begin{tabular}{|c|c|c|c|c|}
\hline $\mathbf{N}^{\circ}$ & Autor & País & Clasificación & Conclusiones \\
\hline & & & & $\begin{array}{l}\text { exige que cada docente cree } \\
\text { ambientes y contextos de } \\
\text { aprendizaje más dinámicos y } \\
\text { flexibles, más cooperativos y } \\
\text { solidarios, la creación de } \\
\text { ecosistemas educacionales en los } \\
\text { cuales predominen la solidaridad, } \\
\text { la asociación, la ética, la } \\
\text { generosidad, el compañerismo, el } \\
\text { diálogo en la búsqueda constante } \\
\text { de soluciones a los conflictos } \\
\text { emergentes, así como el respeto } \\
\text { por las diferencias y el } \\
\text { reconocimiento de la diversidad } \\
\text { cultural y de la existencia de } \\
\text { diferentes estilos de aprendizaje } \\
\text { que enriquecen las experiencias } \\
\text { individuales y colectivas. }\end{array}$ \\
\hline 08 & $\begin{array}{c}\text { Cevallos } \\
\text { (2017). }\end{array}$ & Ecuador & $\begin{array}{l}\text { Artículo } \\
\text { científico }\end{array}$ & $\begin{array}{l}\text { La interdisciplinariedad y la } \\
\text { transdisciplinariedad, son } \\
\text { términos que aún no tienen } \\
\text { claridad en su abordaje e } \\
\text { implicación, sin embargo, ya } \\
\text { forman parte de la estructura del } \\
\text { diseño curricular, del imaginario } \\
\text { de los docentes y de los } \\
\text { requerimientos de la normativa } \\
\text { para su implementación. La } \\
\text { transdisciplinariedad no renuncia } \\
\text { ni rechaza las disciplinas, no } \\
\text { elimina a las disciplinas lo que }\end{array}$ \\
\hline
\end{tabular}


Una mirada reflexiva sobre la $\ldots$

\begin{tabular}{|c|c|c|c|c|}
\hline $\mathbf{N}^{\circ}$ & Autor & País & Clasificación & Conclusiones \\
\hline & & & & $\begin{array}{l}\text { elimina es esa verdad absoluta } \\
\text { que considera que } \\
\text { conocimiento disciplinario es } \\
\text { totalizador, para asumir una } \\
\text { visión sistémica con un enfoque } \\
\text { holístico integrador, transversal, } \\
\text { superando la visión individual y } \\
\text { fragmentada del conocimiento, } \\
\text { rescatando el aporte que cada } \\
\text { disciplina puede dar a esa } \\
\text { construcción del conocimiento y } \\
\text { a la formación del estudiante } \\
\text { como un ser integral. La } \\
\text { transdisciplinariedad } \\
\text { principio epistemológico implica } \\
\text { romper con el asignaturismo } \\
\text { imperante en la educación, ya que } \\
\text { la sociedad del conocimiento } \\
\text { exige hoy una comprensión del } \\
\text { mundo como entidad donde todo } \\
\text { se encuentra entrelazado, } \\
\text { interconectado } \\
\text { interrelacionado. }\end{array}$ \\
\hline 09 & $\begin{array}{c}\text { Espinoza } \\
\text { (2011) }\end{array}$ & México & $\begin{array}{l}\text { Artículo } \\
\text { científico }\end{array}$ & $\begin{array}{l}\text { La transdisciplinariedad es } \\
\text { posible siempre que haya apertura } \\
\text { a nuevas formas de aprender, } \\
\text { renunciando a la certidumbre de } \\
\text { haber alcanzado la verdad y } \\
\text { asumiendo la noción } \\
\text { regenerarse constantemente, este } \\
\text { tránsito es lento y se asienta en la }\end{array}$ \\
\hline
\end{tabular}




\begin{tabular}{|c|c|c|c|c|}
\hline $\mathbf{N}^{\circ}$ & Autor & País & Clasificación & Conclusiones \\
\hline & & & & $\begin{array}{l}\text { disposición, interés, libertad y } \\
\text { voluntad de las personas para } \\
\text { construirlo. La disciplinariedad y } \\
\text { transdisciplinariedad deben } \\
\text { aparecer de manera dialógica, en } \\
\text { los procesos (de formación) } \\
\text { universitarios, como cooperantes } \\
\text { y complementarias, por lo que la } \\
\text { alternancia entre estas se presenta } \\
\text { como necesaria, ya que la } \\
\text { formación universitaria sigue } \\
\text { siendo edificada bajo nociones de } \\
\text { linealidad, reduccionismo, } \\
\text { modelo de ciencia aplicada y } \\
\text { escisión entre sujeto y objeto, } \\
\text { donde el profesor se plantea como } \\
\text { el transmisor del conocimiento. } \\
\text { Finalmente, el acercamiento a la } \\
\text { complejidad y a la } \\
\text { transdisciplinariedad produce un } \\
\text { espacio de libertad para } \\
\text { cuestionar cualquier modelo } \\
\text { socio económico y manifestar } \\
\text { apego o desapego o construir un } \\
\text { esquema social alternativo que } \\
\text { trasciende la noción de desarrollo } \\
\text { tanto social como económico y } \\
\text { permite relaciones más armónicas } \\
\text { naturaleza. }\end{array}$ \\
\hline
\end{tabular}


Una mirada reflexiva sobre la...

\begin{tabular}{|c|c|c|c|c|}
\hline $\mathbf{N}^{\circ}$ & Autor & País & Clasificación & Conclusiones \\
\hline 10 & $\begin{array}{c}\text { Gonzáles } \\
\text { (2014). }\end{array}$ & Perú & $\begin{array}{l}\text { Artículo } \\
\text { Científico }\end{array}$ & $\begin{array}{l}\text { La transdisciplinariedad se } \\
\text { complementa con } \\
\text { interdisciplinariedad, } \\
\text { estudia la realidad de manera más } \\
\text { profunda, integral, global; surge } \\
\text { integrando ciencias para que } \\
\text { respondan a una realidad } \\
\text { compleja, diversa, heterogénea. } \\
\text { Para el sistema educativo } \\
\text { peruano, la transdisciplinariedad, } \\
\text { no solo comprende la integración } \\
\text { de ciencias, sino de sectores } \\
\text { sociales para solucionar los } \\
\text { problemas educativos urgentes, } \\
\text { entre ellos la exclusión educativa, } \\
\text { la segregación social, sobre todo } \\
\text { de las comunidades. Existen otros } \\
\text { trabajos que refieren los retos de } \\
\text { esta disciplina desde su historia, } \\
\text { su etimología, que responda a las } \\
\text { nuevas realidades e incluso a la } \\
\text { misma pedagogía, que debe } \\
\text { responder a la integración } \\
\text { humana, que responda } \\
\text { óptimamente a la formación } \\
\text { humana integral. }\end{array}$ \\
\hline 11 & $\begin{array}{c}\text { Gutiérrez } \\
\text { (2017) }\end{array}$ & México & $\begin{array}{l}\text { Artículo } \\
\text { científico }\end{array}$ & $\begin{array}{l}\text { Con el arribo indetenible de la } \\
\text { ciencia transdisciplinaria, } \\
\text { estamos frente a una generación } \\
\text { que construye conocimiento } \\
\text { libertador, nuevamente libertario, }\end{array}$ \\
\hline
\end{tabular}




\begin{tabular}{|c|c|c|c|c|}
\hline $\mathbf{N}^{\circ}$ & Autor & País & Clasificación & Conclusiones \\
\hline & & & & $\begin{array}{l}\text { no doctrinario, del ciudadano } \\
\text { para el ciudadano, no dogmático } \\
\text { y se puede afirmar que cien años } \\
\text { después de Marx y el marxismo, } \\
\text { que la transdisciplina implica un } \\
\text { brinco epistémico eclético, } \\
\text { entendido más como tolerar la } \\
\text { diferencia, ser inclusivo y } \\
\text { reflexivo y no como una actitud } \\
\text { indecisa o una idea ambigua, } \\
\text { relativista. La gestión pública, la } \\
\text { educación y otras ciencias están } \\
\text { una ruta en la que la ciencia } \\
\text { normal se está volviendo } \\
\text { transdisciplinaria y en la que } \\
\text { todos los caminos llevan al arte de } \\
\text { tener como constante el factor } \\
\text { humano y su condición, diferente } \\
\text { de la ciencia decimonónica, que } \\
\text { separa al arte de la ciencia y a la } \\
\text { religión de ambas. En la } \\
\text { actualidad se está regresando a la } \\
\text { necesidad de alcanzar una } \\
\text { realidad plena al reencontrarse la } \\
\text { ciencia con el arte y el arte con la } \\
\text { ciencia, la religión con la ciencia } \\
\text { y la ciencia con la religión, hay } \\
\text { como un ecunemismo epistémico } \\
\text { de la ciencia de claustro, se } \\
\text { pugnalidad y er la }\end{array}$ \\
\hline
\end{tabular}


Una mirada reflexiva sobre la $\ldots$

\begin{tabular}{|c|c|c|c|c|}
\hline $\mathbf{N}^{\circ}$ & Autor & País & Clasificación & Conclusiones \\
\hline & & & & $\begin{array}{l}\text { entremezclamiento de saberes } \\
\text { vivo y muertos. }\end{array}$ \\
\hline 12 & López (2017) & Venezuela & $\begin{array}{l}\text { Artículo } \\
\text { científico }\end{array}$ & $\begin{array}{l}\text { En la complejidad organizacional } \\
\text { de la nueva gerencia, se enfatiza } \\
\text { las corrientes de pensamiento } \\
\text { complejo concebida desde el } \\
\text { punto de vista de la } \\
\text { transdiciplinariedad, de allí que } \\
\text { las políticas de Estado, la } \\
\text { institucionalidad pública y } \\
\text { privada y la más importante la } \\
\text { participación ciudadana, deben } \\
\text { interactuar sinérgicamente, para } \\
\text { lograr el desarrollo social } \\
\text { sustentable y equitativo para la } \\
\text { comunidad, donde su interacción } \\
\text { con el entorno sea armonioso, } \\
\text { estableciéndose una relación } \\
\text { simbiótica, equilibrada y benéfica } \\
\text { entre la humanidad y el medio } \\
\text { ambiente que provea de } \\
\text { beneficios para ambos. La gestión } \\
\text { pública en la actualidad, debe } \\
\text { caracterizarse por formar } \\
\text { estructuras multidimensionales } \\
\text { que articulen de manera } \\
\text { dialógica, la asociación de varios } \\
\text { aspectos, desde } \\
\text { transdisciplinariedad a } \\
\text { complejidad que realza las }\end{array}$ \\
\hline
\end{tabular}




\begin{tabular}{|l|l|l|l|l|}
\hline $\mathbf{N}^{\circ}$ & Autor & País & Clasificación & \multicolumn{2}{|c|}{ Conclusiones } \\
\hline & & & & $\begin{array}{l}\text { corrientes de pensamiento } \\
\text { complejo. }\end{array}$ \\
\hline
\end{tabular}

\section{Discusión}

Según los artículos revisados, se evidencia que la transdisciplinariedad es poco considerada en el campo de la educación básica ya que tal como lo citamos anteriormente los artículos en su mayoría se refieren a otros campos. En tal sentido queda la tarea de considerar que más allá de producir y aplicar conocimientos científicos específicos, el sistema educativo debe construir la unificación de las ciencias, de saberes, donde se centren los enfoques de la realidad, respecto a la diversidad y libertad filosóficaepistemológica de cada disciplina, sólo así lograremos alcanzar la transdisciplinariedad como propuesta para el avance de la educación. La transdisciplinariedad para el caso de la educación peruana, como lo señala Gonzáles (2014) no solo se trata de integración de ciencias, sino de actores sociales, grupos sociales que respondan a sus propias realidades, necesidades, aspiraciones y no solo de imposiciones que se han realizado a través del Ministerio de Educación que impone un solo currículo, en un solo idioma frente a realidades que hablan en otros idiomas; urge un diálogo intercultural, étnico. Análogamente, en el ámbito de la administración y gestión pública del gobierno; a pesar de la finalidad administrar los recursos del Estado para satisfacer las necesidades de los ciudadanos, muchas de sus políticas públicas no favorecen este cometido, porque su formulación, implementación y evaluación se realizan desde la perspectiva disciplinar del sector asociada al gobierno y no se promueve una gestión participativa que favorezca el diálogo y discusión colectiva con actores de distintas realidades y concepciones. La realidad que cada día percibe el ser humano, la compresión de su propia existencia junto con sus conflictos internos, tiene enorme dosis de complejidad, de lo global, lo contextual y lo multidimensional. Además, la ciencia ha evolucionado, los hallazgos requieren de explicación, además del auge de una nueva forma de pensar y actuar. La humanidad ha experimentado nuevas transformaciones que requieren de una forma de interpretación distinta a la existente por mucho tiempo (Morin, 2004)

En ese sentido, la gestión pública como disciplina de la administración, necesita ser abordada y ejercida con enfoque transdisciplinar por la sencilla razón de administrar y 
gestionar recursos y servicios para una población que vive en determinado territorio, con realidades y problemas complejos como los que afronta la educación. Para Nicolescu, (2002) la visión transdisciplinaria es una perspectiva que propone considerar una realidad multidimensional estructurada en múltiples niveles, que sustituya la visión de una realidad unidimensional del pensamiento conservador; a fin de abordar la solución de los grandes problemas sociales como; la pobreza extrema, la crisis ambiental, la obsolescencia educativa, la crisis de los sistemas de salud, la gobernabilidad social, el desempleo, entre otros que; requieren un tratamiento transdisciplinar porque tienen un nivel de complejidad, interactividad y globalidad, cuya solución según Cándida (2010) rebasa los límites del pensamiento reduccionista y determinista, de planificación positivista y metodología que fragmenta la realidad propios de los modelos de gestión, organización y percepción del siglo XIX.

Por su parte, el enfoque sistémico y transdisciplinar, concibe a la educación como un hecho social orientado a educar al hombre en una realidad muy compleja; a su vez, considera que la educación es un hecho económico, político, jurídico, ambiental, cultural, etc. Entonces, gestionar la educación desde la gestión pública, requiere de cierto nivel de comprensión y el uso de herramientas conceptuales y metodológicas, que recojan el modo de vida dentro de esta perspectiva y tenga en cuenta, el grado de desarrollo de las fuerzas productivas, la organización económica y política, el modo de relacionarnos con el medio ambiente, la cultura, la historia, las relaciones hombre naturaleza y otros procesos que contribuyan a formar la identidad, el sentido de pertenencia, para que la educación favorezca y contribuya verdaderamente al desarrollo integral de los pueblos. En efecto, tanto la educación como la gestión pública debiera nutrirse desde la teoría de la complejidad y la transdisciplinariedad para plantear una verdadera gestión de la educación pública, reestructurando el sistema educativo, reformulando políticas educativas que reflejen y actúen críticamente sobre los nuevos entornos, más sostenibles, resilientes y regenerativos que, trascienda la noción de desarrollo tanto social como económico y permite relaciones más armónicas entre individuo, sociedad y naturaleza (Espinoza, 2011) y que rompa con la unidisciplinariedad imperante en la educación, ya que la sociedad del conocimiento exige hoy una comprensión del mundo como entidad donde todo se encuentra entrelazado, interconectado e interrelacionado (Cevallos, 2017) 


\section{CONCLUSIONES Y CONSIDERACIONES FINALES}

- La educación es un derecho y es una responsabilidad del Estado garantizarlo en el marco de la gestión pública; para ello, resulta necesario dotarle una perspectiva transdisciplinar a la gestión, que articule e integre la convergencias de diferentes teorías y enfoques provenientes de la administración educativa, la sociología de la educación, la economía, la política educativa, la historia de la educación, entre otras con la formación integral del ser humano y el desarrollo del territorio; por cuanto, la educación es un hecho social complejo que rebasa las fronteras de la pedagogía y demás ciencias auxiliares.

- En el proceso de gestionar la educación como un derecho, la administración y gestión pública actual de la mayoría de los gobiernos, casi siempre se encargan de formular, implementar y evaluar políticas públicas dirigidas a la solución de los problemas educativos. Pero, desde la perspectiva de la transdisciplinariedad, lo idóneo es que dichas políticas sean discutidas y definidas desde la óptica de diversas disciplinas que explican los fenómenos sociales asociados a la educación, para que sean fruto de la reflexión y las experiencias de los propios actores en cada contexto, quienes, además se comprometan en la solución de los problemas y finalmente las decisiones sean asumidas fundamentalmente por el Estado y los gobiernos; por cuanto, la complejidad como principio de la transdisciplinariedad exige hoy una comprensión del mundo como entidad donde todo se encuentra entrelazado, interconectado e interrelacionado.

- La transdisciplinariedad y complejidad están estrechamente unidas como formas de pensamiento relacional, y como interpretaciones del conocimiento desde la perspectiva de la vida humana y el compromiso social; por lo tanto, la escuela debe ser considerada como una institución educativa de desarrollo en cada comunidad, cuyos proyectos curriculares y extracurriculares, inter y transdisciplinarios, deben vincularse con el modelo de gestión pública de la región y sus diferentes ejes de desarrollo social, económico, político, ambiental y cultural.

- En la educación básica y superior existen condiciones suficientes para la transdisciplinariedad, pero se requiere una gestión pública que genere las condiciones técnicas, políticas y sociales para encaminar su logro, poniendo como punto inicial, el diálogo de saberes entre los agentes educativos, autoridades académicas y políticas e instituciones de otros sectores, incluidas las que aparentemente no tienen nada 
común entre sí; para hacer realidad el eslogan sobre la educación como estrategia o principal motor de desarrollo y progreso de un país.

\section{LISTA DE REFERENCIAS}

Alfaro Mardones, J. I. Fernández Hernández, C.J. y González García, M. J. (2015). «La transdisciplinariedad una herramienta para apuntar al Buen Vivir», Polis [En línea], Publicado el 18 mayo 2015, consultado el 13 noviembre 2021. URL: http://journals.openedition.org/polis/10896.

Bierbrauer, F. Márquez, M. Una propuesta de trabajo transdisciplinario en el aula, Facultad de Lenguas Universidad Nacional de Córdoba, Córdova Argentina 2013.

Cándida Moraes, María. Transdisciplinariedad y educación, Revista Rizoma Freireano Vol, 06, Instituto Paulo Freire de España. 2010.

Carmona Rodríguez, Miriam A. (2004). Transdisciplinariedad: Una propuesta para la Educación Superior en Venezuela. Revista de Pedagogía,25(73), 59-70. Recuperado en 05 de enero de 2021, de http://ve.scielo.org/scielo.php?script=sci_arttext\&pid=S079897922004000200007\&lng=es\&tlng=es.

Cevallos Mejía, Yasmín Adriana. Transdisciplinariedad y transversalidad. Revista Publicando, 4 No 11. (1). 2017, 499-512. ISSN 1390-9304.Universidad Central del Ecuador.

Espinosa Martínez, Ana Cecilia. (2011) Estrategias metodológicas para operacionalizar la práctica educativa transdisciplinaria, en conjunto con los actores universitarios, en laslicenciaturas del Centro de Estudios Universitarios Arkos (CEUArkos) de Puerto Vallarta, Jalisco, México. Revista Electrónica Educare Vol. XV, № 1, [3156], ISSN: 1409-42-58, Enero-Junio, 2011.

Gedeón Zerpa, Iraida y García Yamín, Nubia. La transdisciplinariedad en la educación superior del siglo XXI. Revista de Artes y Humanidades UNICA Volumen $10 \mathrm{~N}^{\mathrm{o}}$ 3 / Septiembre-Diciembre 2009, pp. 58 - 70 Universidad Católica Cecilio Acosta ISSN: $1317-102 X$.

González Aguilar, Hugo. Inter y transdisciplinariedad: Una reflexión en la educación. Revista PAIDEIA XXI. Vol. 4, Nº 5, Lima, agosto 2014, pp. 95-103.

Gutiérrez de Hoyos, Gustavo. Políticas Públicas y Transdisciplinariedad: Un Paradigma de Futuro para la Demanda Ciudadana. Universidad del Vale de México. 2017. 
Jurado Jiménez, María Dolores. (2013) Investigación autobiográfica en la formación universitaria. Una Metodología que facilita procesos transformadores de educación existencial y social. Universidad de Sevilla.

López Caldera, Mayra (2017). Una mirada reflexiva sobre gestión y salud pública desde el paradigma de la complejidad. Salus, 21 (1), 22-25. [Fecha de Consulta 15 de Noviembre de 2021]. ISSN: 1316-7138. Disponible en: https://www.redalyc.org/articulo.oa?id=375952385005

Martínez Álvarez, Fidel, Ortiz Hernández, Eloy, \& González Mora, Ania. (2007). Hacia una epistemología de la transdisciplinariedad. Humanidades Médicas, 7(2) Recuperado en 15 de noviembre de 2021, de http://scielo.sld.cu/scielo.php?script=sci_arttext\&pid=S1727$81202007000200008 \& \operatorname{lng}=$ es\&tlng=es.

Miguélez, Miguel. Conceptualización de la transdisciplinariedad, Polis, Publicado el 31 julio 2012, consultado el 19 abril 2019. URL : http://journals.openedition.org/ polis/4623.

Morin, Edgar. La epistemología de la complejidad. En: Gazeta de Antropología, № 20, 2004, Artículo 02. Universidad de Granada. http://hdl.handle.net/10481/7253

Nicolescu, Basarab, (2002). Manifiesto de la Transdisciplinariedad. Prensa de la Universidad Estatal de Nueva York, 2002. ISBN $\quad 0791489167$, 978079148916.

Peñuela Velásquez, L. Alejandro. (2005). La transdisciplinariedad: Más allá de los conceptos, la dialéctica. Andamios, 1(2), 43-77. Recuperado en 16 de noviembre de 2021.

http://www.scielo.org.mx/scielo.php?script=sci_arttext\&pid=S1870$00632005000300003 \& \operatorname{lng}=$ es\&tlng=es.

Rodríguez Zoya, Leonardo G. y Leónidas Aguirre, Julio (2011). Teorías de la complejidad y ciencias sociales. Nuevas Estrategias Epistemológicas y Metodológicas. Nómadas. Revista Crítica de Ciencias Sociales y Jurídicas, 30 (2), [Fecha de Consulta 15 de Noviembre de 2021]. ISSN: 1578-6730. Disponible en: https://www.redalyc.org/articulo.oa?id=18120143010. 
Valois Cubillos, N. D. (2015). Integralidad de la gestión desde la transdisciplinariedad. SIGNOS - Investigación En Sistemas De gestión, 7(1), 15-30. https://doi.org/10.15332/s2145-1389.2015.0001.01.

Varona Domínguez, Freddy. (2005). Transdisciplinariedad y educación universitaria. Visión filosófica sobre retos y potencialidades. Universidad de Holguín. Cuba.

Verger, Antoni, \& Normand, Romuald. (2015). Nueva gestión pública y educación: Elementos teóricos y conceptuales para el estudio de un modelo de reforma educativa global. Educação \& Sociedade, 36(132), 599-622. . https://doi.org/10.1590/ES0101-73302015152799. 